\title{
Performance analysis of ultra-wideband system in presence of IEEE802.11a and UMTS/WCDMA frequency bands
}

\begin{abstract}
In order to evaluate the effects of cumulative interference from multiple UWB devices to victim narrowband systems (i.e. IEEE802.11a and UMTS/WCDMA) in its overlay band, an analysis on the power spectral density (PSD) of UWB signal is presented. In this paper, the performances are studied through the bit-error-rate as a function of signal-to-noise ratio as well as signal-to-interference power ratio using computer simulation exploiting realistic channel model (i.e. modified Saleh-Valenzuela (S-V) model). Several modifications of a generic Gaussian pulse waveform with a length in the order of nanoseconds were used to generate UWB spectra. Different kinds of pulse modulation (i.e. antipodal and orthogonal) schemes have been also taken in account.
\end{abstract}

Keyword: Co-existence; FCC; In-band interference; Ultra-wideband; UMTS/WCDMA; WLAN 